\title{
Polyphenol content and in vitro bioaccessibility of six baobab fruit extracts
}

\author{
S. Coe and L. Ryan \\ Functional Foods Centre, Department of Sport and Health Sciences, Oxford Brookes University, Gipsy Lane, \\ Headington, Oxford OX3 OBP, UK
}

The baobab fruit is grown throughout regions in Africa, and is a rich source of soluble fibre and many vitamins and minerals. Anecdotal evidence suggests that baobab may play a role in improving health, yet to date there is little research on the fruit. The health promoting properties are thought to be largely due to the high concentration of polyphenols in baobab fruit. Polyphenols are secondary metabolites found ubiquitously in plants, and are being increasingly recognised as the anti-disease compounds present in many foods. The effect of digestion on polyphenol release is of major interest because the bioaccessibility of polyphenols is thought to change throughout the digestive process. It has been found that antioxidants and phenolics in certain fruit and vegetable juices became more bioaccessible post digestion $^{(1,2)}$. Therefore, the objective of the present study was to analyse the polyphenol content of six baobab fruit extracts. Following this, the bioaccessibility of the polyphenols was evaluated throughout an in vitro digestion procedure.

Six different baobab extracts were used: five from different geographical locations in Africa and one commercial extract purchased in the UK (Holland \& Barret). Total phenols were assessed via the Folin-Ciocalteu method (FCR) ${ }^{(3)}$ and expressed in mg of gallic acid equivalents (GAE) per gram of extract. Total antioxidant capacity was assessed via the ferric-ion reducing antioxidant power assay $(\text { FRAP })^{(4)}$.

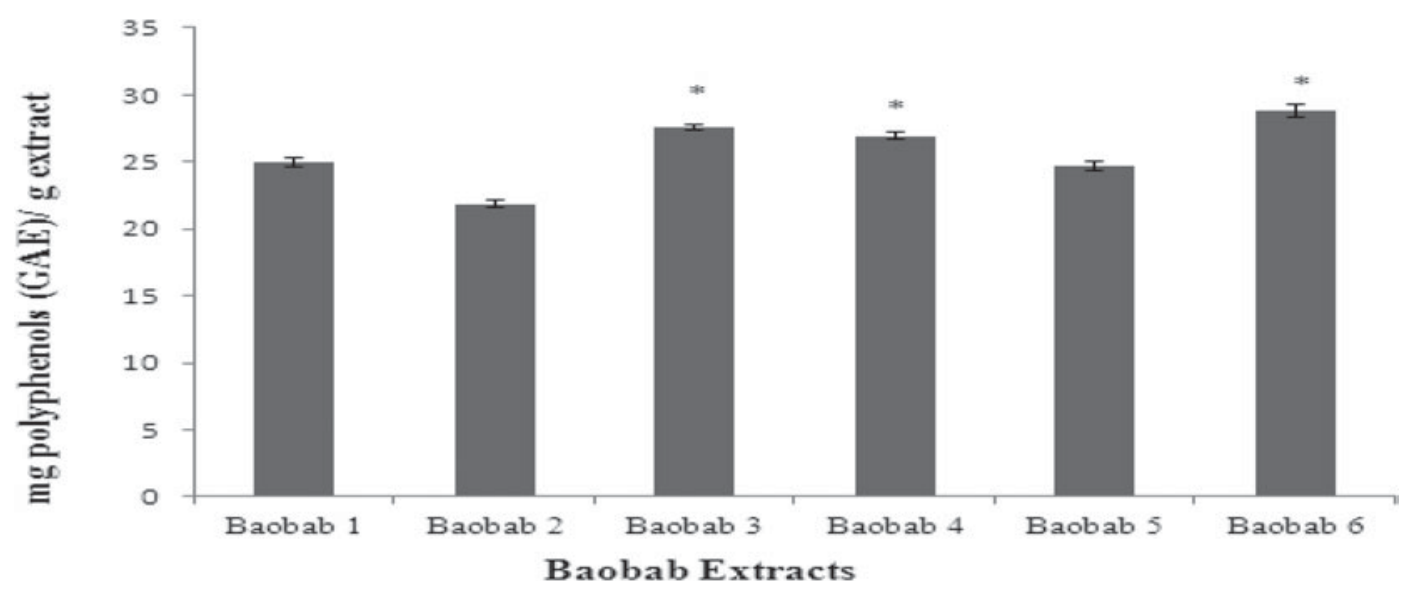

Figure 1: Polyphenol content of 6 baobab extracts. Values are means of 4 independent experiments (ANOVA, followed by Tukey test): $* P<0.05$.

Baobab samples 3, 4 and 6 contained significantly more polyphenols than samples 1,2 and $5(P<0.05)$. Baobab sample 6 (commercial) was shown to have the greatest polyphenol content compared to all other baobab extracts. For all extracts, polyphenol bioaccessibility increased throughout in vitro digestion. FRAP and FCR values were positively correlated at baseline, with FRAP values ranging from $1514-2671 \mu \mathrm{mol} / \mathrm{L}$ and FCR values ranging from $18-34 \mathrm{mg} \mathrm{GAE} / \mathrm{g}$.

All baobab extracts in the study were shown to be good sources of polyphenols and thus show potential as functional food ingredients. FCR values increased after digestion showing that polyphenols in the extracts became more bioaccessible and therefore may potentially be more bioavailable post digestion.

1. Ryan L \& Prescott S (2010) Int J of Food Sci and Tech 45, 1191-1197.

2. Wootton-Beard P, Moran A \& Ryan L (2011) Food Res Int 44, 217-224.

3. Sharma P \& Gujral HS (2010) Food Chem 120, 673-678.

4. Benzie IF \& Strain JJ (1996) Ana Biochem 239, 70-76. 